\title{
The nutrient requirements of Lactobacillus rhamnosus GG and their application to fermented milk
}

\author{
Jiahui Sun, Hongyu Chen, Yali Qiao, Gefei Liu, Cong Leng, Yanjiao Zhang, Xuepeng Lv, and Zhen Feng* \\ Key Laboratory of Dairy Science, Ministry of Education, College of Food Science, Northeast Agricultural University, 600 Changjiang Road, \\ 150030, Harbin, Heilongjiang, China
}

\section{ABSTRACT}

Lactobacillus rhamnosus GG (LGG) performs many physiological functions, but the fermentation time is long when fermented milk is prepared using LGG alone. To shorten the fermentation time, we analyzed the nutrient requirement profiles of LGG. Based on nutrient requirement profiles, we evaluated the effects on the fermentation time, quality, and sensory properties of unmodified cow's milk fermented by LGG alone. According to the consumption and necessary patterns of amino acids and those of purine, pyrimidine, vitamins, metal ions, and nutrients essential to LGG, we selected Cys, Ser, Arg, Pro, Asp, Glu, guanine, uracil, and xanthine with which to supplement milk. Compared with fermented milk prepared using LGG alone in unmodified milk, the fermentation time of supplemented milk was shortened by $5 \mathrm{~h}$. Viable cell counts, titratable acidity, and water-retaining capability of the fermented milk were improved by addition of nutrient supplements. Supplementation with nutrients did not obviously change the sensory and textural characteristics of fermented milk.

Key words: Lactobacillus rhamnosus GG, nutrient requirements, fermented milk, fermentation time, quality

\section{INTRODUCTION}

Lactobacillus rhamnosus Gorbach-Goldin (LGG) is widely used in manufacturing fermented dairy products and other fermented foods. This bacterium exhibits most of the characteristics generally desired for a good probiotic strain, including the ability to survive passage through the human gastrointestinal tract after ingestion and the ability to transiently colonize the ileum and colon (Doron et al., 2005). Additionally, LGG plays a critical role in preventing the colonization of pernicious

Received October 11, 2018.

Accepted March 12, 2019.

*Corresponding author: NEAU_FengZhen@163.com organisms. Strains of L. rhamnosus are usually transient colonizers of the gastrointestinal tract, but strain GG adheres to mucus and epithelial cells (Lebeer et al., 2007; Hou et al., 2016). Frequently, LGG is used as a probiotic, especially in the treatment of small-intestine bacterial overgrowth. Furthermore, LGG has acidic and basic properties and produces exopolysaccharides (Klopper et al., 2017).

The health-beneficial properties of LGG depend in part on its prolonged residence in the gastrointestinal tract and strong adhesive capacity. Therefore, LGG is an excellent starter for fermented milk manufacturing, and milk fermented with LGG has many probiotic functions for adults and children. This bacterium has the advantages of resisting low $\mathrm{pH}$ levels and has an excellent shelf life (Sharareh et al., 2009). It is also a possible candidate for synthesis of acetoin and diacetyl, which are butter-like flavor compounds that are extensively used in the dairy industry (Jyoti et al., 2004). However, most probiotics, including L. rhamnosus strains, grow slowly in milk, and the rate of acid production is usually too slow to support an adequate fermentation process (Shah, 2000). The fermentation time of milk prepared using LGG alone is long, and most commercial probiotics incorporated in dairy products include strains belonging to the genera Lactobacillus, Lactococcus, and Bifidobacterium (Lourens-Hattingh and Viljoen, 2001). A long fermentation time is unfavorable to the industrialization of fermented milk prepared using a single probiotic strain. At present, many fermented dairy products on the market are prepared using a single probiotic strain, such as Yakult (Lactobacillus casei strain Shirota, Yakult, Guangzhou, China), LAJOIE (Lactobacillus paracasei LPC-37, Wanjiabao Dairy Co., Ltd, Harbin, China), and Cherita (L. rhamnosus GG ATCC 53103, Hebei Fermented Biotechnology Co., Ltd., Beijing, China). The viable cell counts, which are important for performing physiological functions, of fermented dairy products using a single probiotic strain are far greater than those using mixed strains. Fermented dairy products prepared using a single probiotic strain have long fermentation times (Byung-Ju 
et al., 2005). A long fermentation time also limits the use of LGG in fermented dairy products and other fermented foods. Therefore, shortening the fermentation time of milk fermented using LGG alone should be considered.

The aim of the present study was to shorten the fermentation time of milk prepared with LGG alone by adding nutrients to the milk based on the specific nutritional requirements of LGG. Furthermore, we evaluated the effects on the quality and sensory properties of fermented milk prepared with LGG alone, in supplemented and unsupplemented milk.

\section{MATERIALS AND METHODS}

\section{Strain, Culture Conditions, and Fermentation Experiments}

Strain ATCC 53103 of LGG was acquired from the American Type Culture Collection (Manassas, VA). Cultures were stored at $-80^{\circ} \mathrm{C}$ in $15 \%$ sterile reconstituted skim milk containing $10 \%$ glycerol. Eight subcultures were prepared in chemically defined medium $(\mathbf{C D M})$ for $12 \mathrm{~h}$ at $37^{\circ} \mathrm{C}$. The $\mathrm{CDM}$ was prepared according to the method of Savijoki et al. (2006). The cells from the 8 subcultures were harvested by centrifugation $\left(10,000 \times g, 5 \mathrm{~min}, 4^{\circ} \mathrm{C}\right)$. The pellet was washed twice with PBS (50 mmol/L, $\mathrm{pH} 6.5)$, and the culture was inoculated in a 10-L Biotech-7000 bioreactor (Shanghai Baoxing, Shanghai, China) containing 7 $\mathrm{L}$ of CDM. The temperature and rotation speed were fixed at $37^{\circ} \mathrm{C}$ and $150 \mathrm{rpm}$, respectively. The $\mathrm{pH}$ was initially set at 6.6 in uncontrolled $\mathrm{pH}$ cultures. Samples were harvested at $0,3,7,10$, and $12 \mathrm{~h}(\mathrm{t} 0, \mathrm{t} 1, \mathrm{t} 2, \mathrm{t} 3$, and $\mathrm{t} 4$ as shown in Figure 1a). The culture supernatant and cells were obtained by centrifugation $(10,000 \times g, 5$ min, $4^{\circ} \mathrm{C}$ ) and preserved at $-80^{\circ} \mathrm{C}$ for further analysis. Consumption profiles of the nutrients were obtained through analysis of the culture supernatants after centrifugation at the aforementioned growth times. Batch fermentations were repeated 3 times.

\section{Analysis of AA, Vitamins, Purines, Pyrimidine, and lons}

To measure whole-cell AA composition and determine the required amounts of each $\mathrm{AA}$, the biomass was hydrolyzed with $6 \mathrm{~mol} / \mathrm{L}$ of $\mathrm{HCl}$ for $20 \mathrm{~h}$ at $120^{\circ} \mathrm{C}$. Amino acids were measured by an amino acid analyzer (Acquity UPLC; Waters Corp., Milford, MA). Concentrations of folic acid, riboflavin, calcium pantothenate, biotin, pyridoxine, thiamin- $\mathrm{HCl}$, myo-inositol, and cyanocobalamin were determined by reverse-phase (RP)-HPLC with a UV detector (Heudi et al., 2005).
$P$-Aminobenzoic acid and nicotinic acid concentrations were determined by HPLC and RP-HPLC, respectively (Okdeh et al., 2003; Ciulu et al., 2011). Concentrations of adenine, guanine, xanthine, and uracil were measured by liquid chromatography (LC)-MS/MS (Lorenzetti et al., 2007; Stentoft et al., 2014). Ion concentrations were determined using inductively coupled plasma-atomic emission spectrometry (Tu et al., 2013). Essential, nonessential, and stimulatory nutrients were determined through single-omission experiments (Vera Pingitore et al., 2009); briefly, we studied the relationship between medium components and growth when a constituent was omitted from the medium.

\section{Selection of Nutrients}

The consumption and necessary patterns of nutrients, and essential nutrients for the growth of LGG, and the concentrations of nutrients in unmodified milk were analyzed and compared. The nutrients with high consumption and necessary rates and essential nutrients for growth of LGG were added to milk to conduct the preliminary single-addition experiments. The aim of the preliminary single-addition experiments was to select those nutrients that reduced the fermentation time of milk prepared with LGG. Each of these chosen nutrients was added to milk for manufacturing the fermented milk.

\section{Preparation of Fermented Milk}

We inoculated and propagated LGG 3 times in 12\% (wt/wt) sterile reconstituted skim cow milk at $37^{\circ} \mathrm{C}$. The mother culture was prepared in $12 \%$ (wt/wt) reconstituted skim milk. To prepare fermented milk, the milk was filtered before the addition of glucose $(4.6 \%$, wt/wt). The milk was pasteurized at $95^{\circ} \mathrm{C}$ for $5 \mathrm{~min}$ and cooled to $37^{\circ} \mathrm{C}$. The mother culture was inoculated into the milk at $2 \%(\mathrm{vol} / \mathrm{vol})$, incubated at $37^{\circ} \mathrm{C}$ until the $\mathrm{pH}$ reached 4.5 , and then cooled to $4^{\circ} \mathrm{C}$. According to the nutrients selected, fermented milks were prepared with the addition of Cys, Ser, Arg, Pro, Asp, Glu, guanine, uracil, and xanthine to the milk. A total of $200 \mathrm{~kg}$ of fermented milk was manufactured from each fermentation batch. Each batch was prepared with unmodified milk (100 kg) or with modified milk (100 $\mathrm{kg}$ ). Batch fermentations were repeated 3 times.

\section{Physicochemical, Microbiological, Sensory, and Textural Analyses of Fermented Milk}

Water-holding capacity and titratable acidity were measured as described by Jia et al. (2016). Textural properties (hardness, adhesiveness, springiness, and 

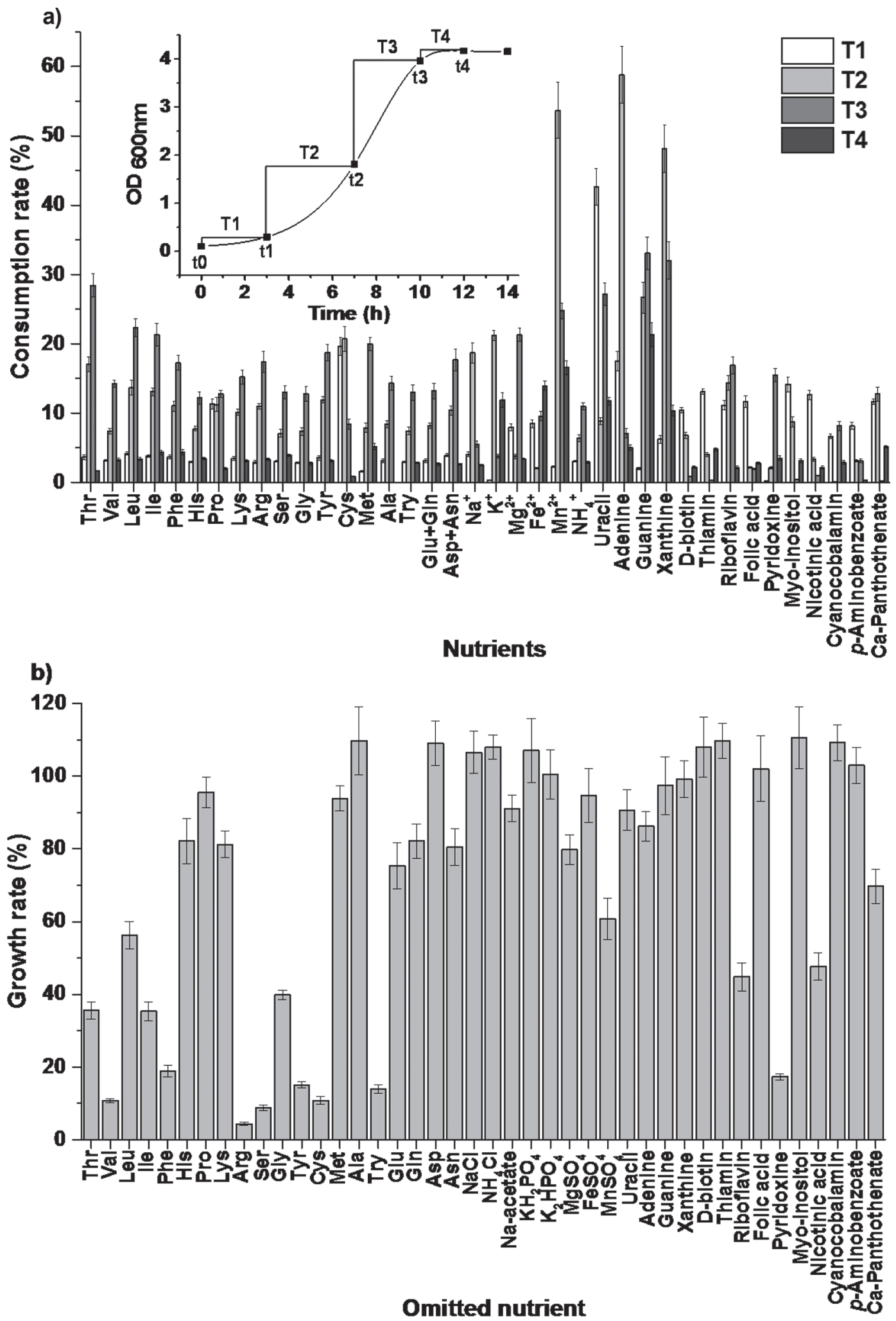

Figure 1. (a) Nutrient consumption patterns of Lactobacillus rhamnosus GG (LGG) at different average growth rates [T1, T2, T3, and T4; inset, measured as optical density (OD) at $600 \mathrm{~nm}$. The values are the ratio (\%) of the consumption amount in each stage to the initial addition amount. (b) Effects of nutrient omission on the growth rate of LGG. Growth rates were expressed as the percentage of the growth rate when a nutrient was omitted from the chemically defined medium (CDM) compared with that in complete CDM. Error bars represent SE. 
cohesiveness) were analyzed with compression tests using a Texture Analyzer TA-Plus texturometer (Lloyd Instruments, Bognor Regis, UK), as described by Miocinovic et al. (2016). The sensory properties of fermented milk were evaluated as described by Ahtesh et al. (2017). Viable cell counts were evaluated using the standard dilution method in de Man, Rogosa, and Sharpe agar medium (HuanKai Microbial Sci. \& Tech. Co., Ltd., Guangdong, China).

\section{Statistical Analysis}

Statistical analyses were conducted using Statistix 8.1 (Analytical Software, Tallahassee, FL). All data were expressed as mean values with standard deviation.

\section{RESULTS}

\section{Nutrient Consumption Profiles}

According to the concentrations of the nutrients in the CDM, we determined the consumption profiles of the nutrients (Figure 1a). Figure 1a shows the ratio of the consumed amount to the initial addition amount in each stage. The ratio of total consumption is the sum of the consumption ratios of each growth stage. For the consumption patterns of AA, Thr $(50.77 \%)$ and Cys (49.60\%) were consumed the most during the growth of LGG. Arginine, Ile, Phe, Tyr, Leu, Pro, Lys, Asp, Asn, and Met ranked as the next most-consumed AA, exceeding $30 \%$ of their initial concentrations and, in the case of Ile and Leu, surpassing $40 \%$ of the initial concentrations. For consumption profiles of other nutrients, over $50 \%$ of the initial concentration of adenine, guanine, uracil, and xanthine was consumed. In particular, the consumption of adenine reached $88.47 \%$ of its initial concentration. The consumption of riboflavin was the most intensive among the vitamins, reaching $44.55 \%$ of its initial concentration. Consumption of $\mathrm{Mn}^{2+}$ was $97.34 \%$, making it the most-consumed metal ion.

\section{Effect of Nutrients on the Growth of LGG}

Based on single-omission experiments, required nutrients, stimulatory nutrients, and nonrequired nutrients were determined (Figure 1b). A nutrient was considered essential if its omission led to growth rates of less than half the maximum growth rate of the positive control, stimulatory when in its absence the growth rate was between 50 and $80 \%$ of that observed in complete CDM, and nonessential when the growth rate in its absence was $80 \%$ (or more) of that obtained in the complete CDM. The results showed that Arg, Val, Ile, Phe, Thr, Ser, Gly, Try, Tyr, and Cys were essential for the growth of LGG. Leucine, Glu, calcium pantothenate, $\mathrm{Mn}^{2+}$, and $\mathrm{Mg}^{2+}$ had stimulatory effects on the growth of LGG, and the removal of other nutrients had no obvious effects on the growth of LGG.

\section{Profiles of $A A$}

We calculated the required amounts of AA according to their concentrations in the cells (Figure 2a). Figure 2 a shows the ratio of the required amount of individual to total AA. The required amount of Val accounted for $15.59 \%$ of the total AA requirements, higher than the amount of other AA needed for growth of LGG. The required amounts of Glu, Phe, and Asp were next, at 9.60, 8.81, and 7.9\%, respectively, followed by Ala, Met, Tyr, Lys, Cys, and Leu, which ranged from 5 to $7 \%$ of all AA required.

\section{Nutrient Selection}

A comparison of the present results with the free AA concentrations and the concentrations of other nutrients in milk showed that some nutrients needed for growth of LGG are lacking in cow milk (Table 1). According to the consumption patterns of nutrients and their concentrations in milk, AA and vitamins with high consumption ratios (more than $30 \%$ ) should be added to milk. Other nutrients' consumption ratios were also greater than 50\%; thus, Cys, Arg, Ile, Phe, Thr, Tyr, Leu, Pro, Lys, Asp, Asn, Met, adenine, guanine, uracil, xanthine, and riboflavin were added to milk to conduct the preliminary single-addition experiments. According to the profiles of AA and their concentrations in milk, AA with high requirement ratios (close to or more than $8 \%$ ) should also be added. According to the AA requirement amount, the required amount of AA less than $8 \%$ did not improve the growth of LGG. Therefore, Val and Glu were added to milk for the preliminary single-addition experiments. In addition, according to the essential nutrients for growth of LGG and their concentrations in milk, Ser and Gly were also added for the preliminary single-addition experiments.

Single-addition experiments tested the effects of the aforementioned nutrients on the fermentation time of fermented milk prepared using LGG. Figure $2 \mathrm{~b}$ shows nutrients that promote fermentation. The results showed that the growth-promoting effects of most of the nutrients on the fermentation process occurred mainly in the early stage of fermentation. Compared with control samples, fermentation time was shortened by $2 \mathrm{~h}$ or more, and the nutrients were selected to be added to milk in combination. According to the present results, Cys, Arg, Pro, Asp, Ser, Glu, guanine, uracil, and xanthine were selected for addition of milk 

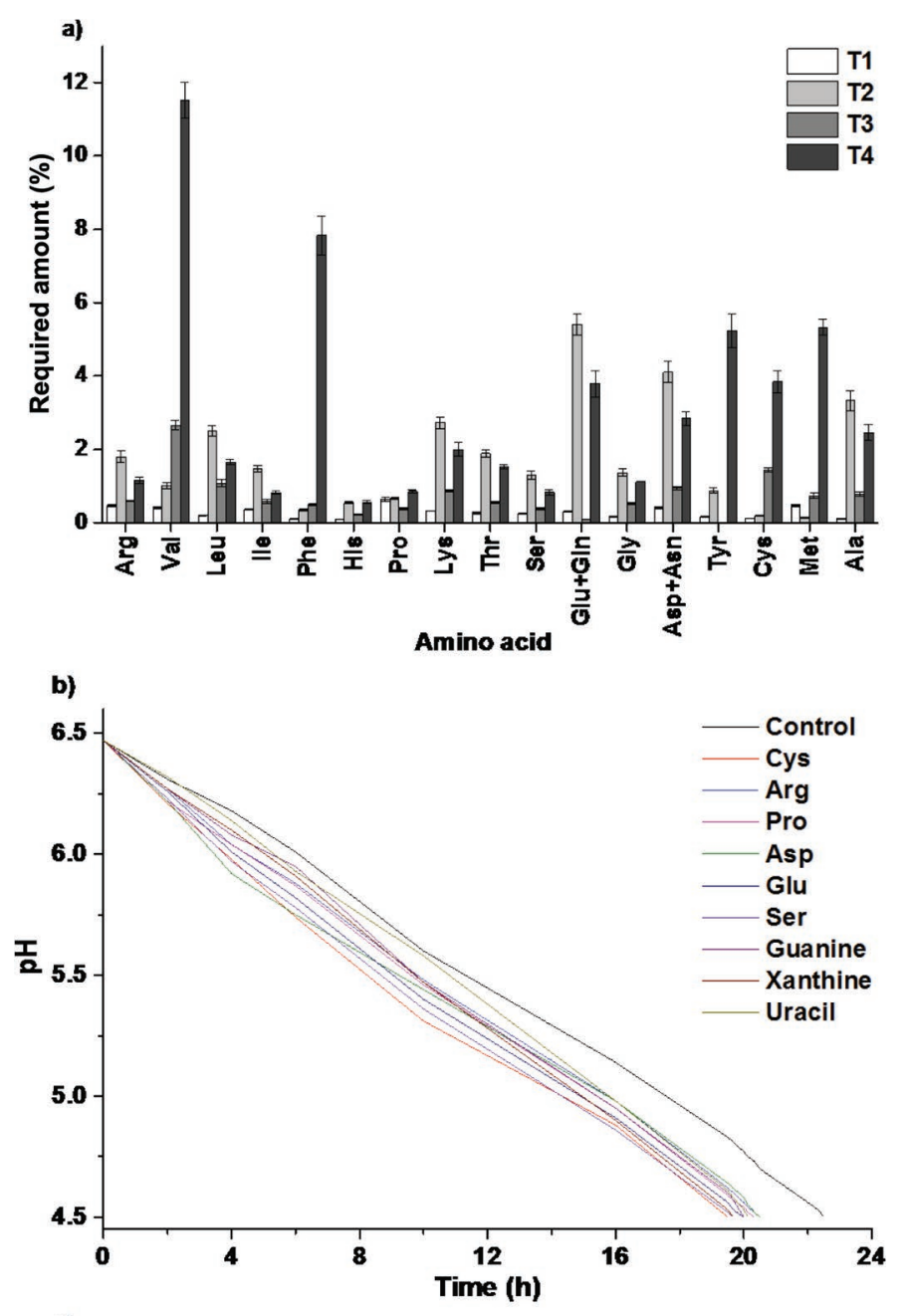

c)

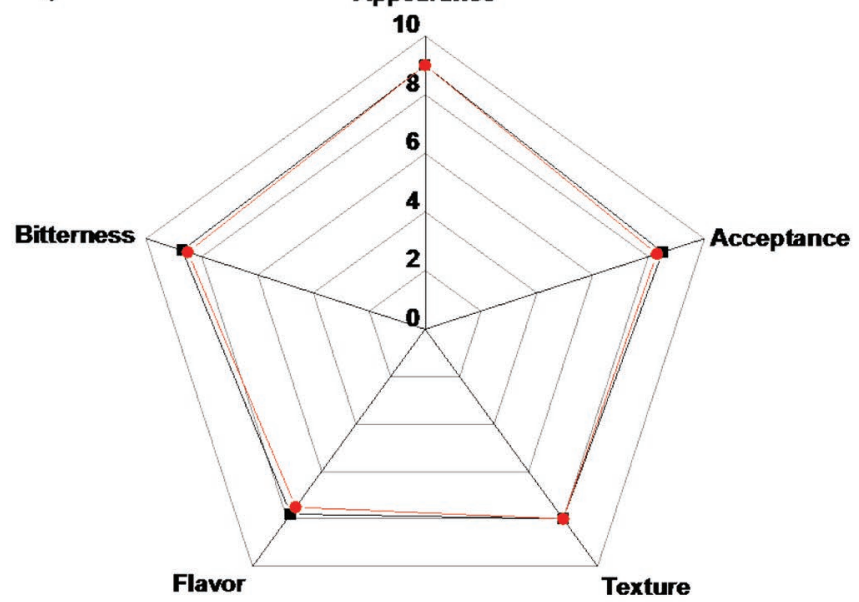

- - Control fermented milk sample - - Modified fermented milk sample

Figure 2. (a) Required amounts (\%) of AA for growth of Lactobacillus rhamnosus GG (LGG) at different average growth rates (T1, T2, T3, and T4, in Figure 1). Error bars represent SE. (b) Effects of nutrients that promote fermentation in the LGG strain on $\mathrm{pH}$ of culture over time. (c) Graphical representation of the mean sensory evaluation by quantitative descriptive analysis of control and modified (supplemented) fermented milk samples. together, based on their consumption and requirement amounts. The concentrations of the selected nutrients are shown in Table 1.

\section{Effects of Supplemental Nutrients on the Physicochemical, Microbiological, Textural, and Sensory Properties of Fermented Milk}

The effects of the selected nutrients on fermentation time, viable cell count, water-retaining capability, titratable acidity, textural properties, and sensory properties of fermented milk prepared with LGG were evaluated. The results are shown in Table 2 and Figure 2c. Compared with the unmodified milk sample, the fermentation time of milk prepared using LGG in milk supplemented with nutrients was shortened by approximately $5 \mathrm{~h}$. The viable cell counts of fermented milk prepared using LGG reached $10^{9} \mathrm{cfu} / \mathrm{mL}$ in the milk supplemented with nutrients, twice that of those in the unmodified milk sample. The differences in appearance, acceptability, flavor, and texture were not obvious between fermented milk from the unmodified milk samples and that from the modified milk samples. Two panelists believed that the bitterness score of fermented milk prepared with LGG using modified milk was slightly higher than that of the unmodified milk samples.

Sensory properties of the fermented milk were assessed by 20 trained panelists recruited from staff members and students from the Northeast Agricultural University. Panelists were selected according to international standards (ISO, 2012). The lighting and environmental conditions for the test were in accordance with international standards (ISO, 2007). Samples in 30-mL white plastic cups coded with 1 digit at room temperature were presented to each panelist. Water and crackers were given to panelists for palate cleansing between samples, allowing 15-min breaks between sessions. Panelists were advised not to swallow the product. Each panelist evaluated 2 samples for flavor, bitterness, texture, appearance, and acceptance, using a 10 -point hedonic scale $(1=$ dislike extremely to $10=$ like extremely). This was not a statistically significant finding.

The average scores of appearance were 9 in the modified fermented milk and the control fermented milk. The average scores of texture were 8 in the modified fermented milk and the control fermented milk. The average scores of flavor were 7.5 in the modified fermented milk, and 7.8 in the control fermented milk. The average scores of bitterness were 8.5 in the modified fermented milk, and 8.7 in the control fermented milk. The average scores of acceptance were 8.3 in the 
modified fermented milk, and 8.5 in the control fermented milk.

\section{DISCUSSION}

A shortage of nutrients is the main limiting factor for the growth of lactic acid bacteria (Youssef et al., 2005). Correcting the nutrient-limiting conditions could be an effective way to improve the fermentation process. Although the growth of heterolactic lactic acid bacteria strains has been studied under various conditions, including variations in $\mathrm{pH}$ and in concentrations of initial substrates and lactic acid, only a few studies have provided a detailed analysis of nutritional medium limitations from a kinetic perspective. The present study highlights the requirements of certain nutrients for the growth of LGG. If these substances are supplied at an insufficient level, efficient growth of LGG might not be achieved. Furthermore, nutrients with high consumption rates and their necessary amounts and essential nutrients should be considered in fermented foods prepared using LGG, to shorten the fermentation time.

The addition of Cys, Arg, Pro, Asp, Ser, Glu, guanine, uracil, and xanthine to milk shortened the fermentation time of the milk prepared with LGG. Based on the L. rhamnosus genome, metabolic maps of Cys, Arg, Pro, Asp, Ser, Glu, guanine, uracil, and xanthine have been predicted (Morita et al., 2009). Lactobacillus rhamnosus can synthesize Cys, Arg, Pro, guanine, and xanthine but not uracil. Cysteine, Arg, and Pro can be synthesized from Ser and Glu, but L. rhamnosus cannot synthesize Glu and Ser through a de novo pathway. Guanine and xanthine were synthesized from guanosine and xanthosine, but L. rhamnosus does cannot synthesize guanine and xanthine through a de novo pathway. The substrates for synthesis of these nutrients were deficient in milk or synthesis rates of these nutrients did not meet the growth needs of LGG. These nutrients were important to the growth of LGG and reduced the fermentation time of milk prepared with LGG.

Most nutrients promoted fermentation of milk primarily in the early stage of the fermentation process $(0-8 \mathrm{~h})$. We can infer that they are likely to promote the metabolism and growth of LGG in the lag growth phase and early exponential growth phase, as a stimulating factor. Most nutrients promoted fermentation of milk primarily in the early stage of the fermentation process $(0-8 \mathrm{~h})$. Furthermore, we can infer that LGG can hydrolyze milk proteins to obtain the AA needed and can synthesize other nutrients needed in later in the fermentation process, but this ability is weak in the early stage of fermentation. Therefore, most nutrients only increased the start-up speed of LGG and shortened the lag growth phase in milk. The reduction of fermentation time is attributable to the improved rate of acid production by LGG from the lag phase to the mid-exponential growth phase. In addition, flavor and aroma are important properties for consumer acceptance. Some panelists found the bitterness score of fermented milk prepared using LGG in modified milk to be slightly higher than that of unmodified milk samples (result was not significant). The addition of AA might

Table 1. Total consumption amount of nutrients, required amounts of AA, and their additive amounts in milk

\begin{tabular}{llccc}
\hline Nutrient & $\begin{array}{c}\text { Milk } \\
(\mathrm{g} / \mathrm{L})\end{array}$ & $\begin{array}{c}\text { Consumption } \\
(\mathrm{g} / \mathrm{L})\end{array}$ & $\begin{array}{c}\text { Requirement } \\
(\mathrm{g} / \mathrm{L})\end{array}$ & $\begin{array}{c}\text { Addition } \\
(\mathrm{g} / \mathrm{L})\end{array}$ \\
\hline Cys & 0 & 0.6088 & 0.3895 & 0.6088 \\
Arg & 0.000551 & 0.1281 & 0.2798 & 0.2798 \\
Ile & 0.000164 & 0.2680 & 0.2246 & 0.2680 \\
Phe & 0.000148 & 0.2013 & 0.6119 & 0.6119 \\
Thr & 0.000285 & 0.4243 & 0.2946 & 0.4243 \\
Tyr & 0.001305 & 0.1731 & 0.4372 & 0.4372 \\
Leu & 0.000961 & 0.3016 & 0.3791 & 0.3791 \\
Pro & 0 & 0.0956 & 0.1776 & 0.1776 \\
Lys & 0.003115 & 0.3526 & 0.4230 & 0.4230 \\
Asp & 0 & 0.3319 & 0.5493 & 0.5493 \\
Asn & 0 & 0.3319 & 0.5493 & 0.5493 \\
Met & 0.000211 & 0.0714 & 0.4634 & 0.4634 \\
Val & 0.003751 & 0.2287 & 0.687 & 1.084 \\
Glu & 0.05126 & 0.4060 & 0.1915 & 0.6674 \\
Ser & 0.000257 & 0.4387 & 0.6867 & 0.4387 \\
Gly & 0.000419 & 0.1190 & -1 & 0.6867 \\
Adenine & 0.002728 & 0.0086 & - & 0.0086 \\
Guanine & - & 0.0077 & - & 0.0077 \\
Xanthine & - & 0.0070 & - & 0.0070 \\
Uracil & - & 0.0042 & & 0.0042 \\
Riboflavin & 0.00175 & 0.0023 & & 0.0023 \\
\hline
\end{tabular}

$1 \_=$undetected. 
have led to this result (Ahtesh et al., 2017). Panelists did not detect any significant difference in aroma between the fermented milk samples from unmodified milk and modified milk. The results indicate that the addition of nutrients had no adverse effect on the quality of fermented milk.

\section{CONCLUSIONS}

This work integrated the nutrient limitations and requirements of LGG and of milk fermented using LGG. Our results indicated that the nutrients Cys, Arg, Pro, Asp, Ser, Glu, guanine, uracil, and xanthine, which were supplemented to the milk prepared with LGG, shortened the fermentation time by $5 \mathrm{~h}$. The addition of these nutrients did not affect the textural or sensory properties, or flavor compounds of the fermented milk. The viable cell counts, water-retaining capability, and titratable acidity of the fermented milk prepared with LGG in the modified milk were increased. We designed a new milk medium for fermented milk prepared solely with LGG, by adding specific nutrients, and the resultant probiotic fermented milk had good properties and a shorter fermentation time.

\section{ACKNOWLEDGMENTS}

This work was supported by grants from the National Key Research and Development Plan Project (2018YFD0400405), National Natural Science Foundation of China (31771989) and Natural Science Foundation of Heilongjiang Province (C2016023).

\section{REFERENCES}

Ahtesh, F. B., L. Stojanovska, and V. Apostolopoulos. 2018. Processing and sensory characteristics of a fermented low-fat skim milk drink containing bioactive antihypertensive peptides, a functional milk product. Int. J. Dairy Technol. 71:230-239.

Byung-Ju, J., S. Jin-Seok, and K. Hae-Soo. 2005. Physico-chemical properties of Lactobacillus casei 00692 during fermenting for liquid-type yogurt. Korean J. Food Sci. Anim. Resour. 25:226-231.

Ciulu, M., S. Solinas, I. Floris, A. Panzanelli, M. I. Pilo, P. C. Piu, N. Spano, and G. Sanna. 2011. RP-HPLC determination of watersoluble vitamins in honey. Talanta 83:924-929.

Doron, S., D. R. Snydman, and S. L. Gorbach. 2005. Lactobacillus GG: Bacteriology and clinical applications. Gastroenterol. Clin. North Am. 34:483-498.

Heudi, O., T. Kilinç, and P. Fontannaz. 2005. Separation of watersoluble vitamins by reversed-phase high performance liquid chromatography with ultra-violet detection: Application to polyvitaminated premixes. J. Chromatogr. A 1070:49-56.

Hou, C., X. Zeng, F. Yang, H. Liu, and S. Qiao. 2015. Study and use of the probiotic Lactobacillus reuteri in pigs: A review. J. Anim. Sci. Biotechnol. 6:14.

ISO. 2007. ISO 8589. Sensory analysis - General guidance for the design of test rooms. International Organization for Standardization, Geneva, Switzerland.

ISO. 2012. ISO 8586. Sensory analysis-General guidelines for the selection, training and monitoring of selected assessors and expert 
sensory assessors. International Organization for Standardization, Geneva, Switzerland.

Jia, R., H. Chen, H. Chen, and W. Ding. 2016. Effects of fermentation with Lactobacillus rhamnosus GG on product quality and fatty acids of goat milk yogurt. J. Dairy Sci. 99:221-227.

Jyoti, B. D., A. K. Suresh, and K. V. Venkatesh. 2004. Effect of preculturing conditions on growth of Lactobacillus rhamnosus, on medium containing glucose and citrate. Microbiol. Res. 159:35-42.

Klopper, K. B., S. M. Deane, and L. M. T. Dicks. 2018. Aciduric strains of Lactobacillus reuteri, and Lactobacillus rhamnosus, isolated from human feces, have strong adhesion and aggregation properties. Probiotics Antimicrob. Proteins. 10:89-97.

Lebeer, S., T. L. Verhoeven, M. Perea Vélez, J. Vanderleyden, and S. C. De Keersmaecker. 2007. Impact of environmental and genetic factors on biofilm formation by the probiotic strain Lactobacillus rhamnosus GG. Appl. Environ. Microbiol. 73:6768-6775.

Lorenzetti, R., S. Lilla, J. L. Donato, and G. de Nucci. 2007. Simultaneous quantification of GMP, AMP, cyclic GMP and cyclic AMP by liquid chromatography coupled to tandem mass spectrometry. J. Chromatogr. B Analyt. Technol. Biomed. Life Sci. 859:37-41.

Lourens-Hattingh, A., and B. C. Viljoen. 2001. Yogurt as probiotic carrier food. Int. Dairy J. 11:1-17.

Miocinovic, J., Z. Miloradovic, M. Josipovic, A. Nedeljkovic, M. Radovanovic, and P. Pudja. 2016. Rheological and textural properties of goat and cow milk set type yoghurts. Int. Dairy J. 58:43-45.

Morita, H., H. Toh, K. Oshima, M. Murakami, T. D. Taylor, S. Igimi, and M. Hattori. 2009. Complete genome sequence of the probiotic Lactobacillus rhamnosus ATCC 53103. J. Bacteriol. 191:7630-7631.

Okdeh, M. S., S. Mostafa, and S. Traboulssie. 2003. Determination of p-amino benzoic acid and p-hydroxy methyl benzoate in sunscreens by HPLC. Asian J. Chem. 15:667-674.
Savijoki, K., A. Suokko, A. Palva, and P. Varmanen. 2006. New convenient defined media for $[(35) \mathrm{S}]$ methionine labelling and proteomic analyses of probiotic lactobacilli. Lett. Appl. Microbiol. 42:202-209.

Shah, N. P. 2000. Probiotic bacteria: Selective enumeration and survival in dairy foods. J. Dairy Sci. 83:894-907.

Sharareh, H., H. Soltani, and G. Reid. 2009. Growth and survival of Lactobacillus reuteri $\mathrm{RC}-14$ and Lactobacillus rhamnosus GR-1 in yogurt for use as a functional food. Innov. Food Sci. Emerg. Technol. 10:293-296.

Stentoft, C., M. Vestergaard, P. Løvendahl, N. B. Kristensen, J. M. Moorby, and S. K. Jensen. 2014. Simultaneous quantification of purine and pyrimidine bases, nucleosides and their degradation products in bovine blood plasma by high performance liquid chromatography tandem mass spectrometry. J. Chromatogr. A 1356:197-210.

Tu, Y. G., Y. Zhao, and M. S. Xu. 2013. Simultaneous determination of 20 inorganic elements in preserved egg prepared with different metal ions by ICP-AES. Food Anal. Methods 6:667-676.

Vera Pingitore, E., E. M. Hebert, F. Sesma, and M. E. Nader-Macías. 2009. Influence of vitamins and osmolites on growth and bacteriocin production by Lactobacillus salivarius CRL 1328 in a chemically defined medium. Can. J. Microbiol. 55:304-310.

Youssef, C. B., G. Goma, and A. Olmos-Dichara. 2005. Kinetic modelling of Lactobacillus casei ssp. rhamnosus growth and lactic acid production in batch cultures under various medium conditions. Biotechnol. Lett. 27:1785-1789. 